\title{
Legal Thinking on the Participation of Traditional Chinese Medicine in Public Health Emergencies
}

\author{
Xinghua Chen \\ Ph.D., Associate Professor, North China University of Technology \\ (Beijing, China) \\ E-mail: xinghuachen@126.com \\ https://orcid.org/0000-0003-1189-7784
}

Chen, Xinghua (2020) Legal Thinking on the Participation of Traditional Chinese Medicine in Public Health Emergencies. Ukrainian Policymaker, Volume 6, 6-14. https://doi.org/10.29202/ up/6/1

The healing effect of traditional Chinese medicine in the epidemic situation with COVID-19 is increasingly confirmed. In a medical system dominated by Western medicine, it is necessary to overcome many obstacles to the participation of traditional Chinese medicine in responding to public health emergencies. This includes (a) natural institutional difficulties; (b) insufficient regulatory ability of traditional Chinese medicine and lack of provisions Traditional Chinese emergency medicine of the public health legal system; (c) the lack of a rational understanding of traditional medicine by the population; and (d) the dilemma of the practice of traditional Chinese doctors. To fully appreciate the role of traditional Chinese medicine in responding to public health emergencies, it is necessary (a) to improve the relevant public health emergency laws; (b) improve the status of local branches of traditional Chinese medicine; (c) strengthen advocacy for the development of traditional Chinese medicine; (d) expand the areas of traditional Chinese medicine in public health emergency management, as well as the openness of traditional Chinese traditional medicine in public health emergencies.

Keywords: traditional chinese medicine, public health emergencies, COVID-19, emergency legal system

Received: May 12, 2020; accepted: June 1, 2020

(C) Chen, Xinghua, 2020 


\section{Introduction}

The COVID-19 epidemic overwhelmed in China at the beginning of 2020. On January 23, Wuhan City announced an unprecedented decision of lockdown. Wuhan, a hero city, began to fight against an arduous war of "epidemic." In this war against the COVID-19 epidemic, hospitals are battlefields, and medical staffs are soldiers. Currently, the universal medical system determines the dominant role of western medicine in response to public health emergencies around the world.

To some extent, traditional Chinese medicine (TCM), which has a long history of over 1000 years, has been developing and perfecting continuously based on fights against epidemics (Liang, 2020: 6). How is the cooperation of TCM and western medicine in this COVID-19 epidemic war in Wuhan? China has achieved outstanding progress in this fight since March. However, COVID-19 epidemic began to spread quickly in other countries, firstly in European countries like Italy. Virus is the enemy of the entire mankind. The curative effect of Chinese treasure - TCM in fighting against the COVID-19 epidemic is more and more confirmed. This study is going to investigate barriers against the participation of TCM in response system to public health emergencies as well as the relevant perfection pathways, aiming to make full use of TCM in epidemics.

\section{Foreword}

Based on performances in SARS epidemic control, the important role of TCM in response to public health emergencies has been confirmed from the perspective of governments. On February 12, 2020, the General Office of National Health Commission of the People's Republic of China and Office of National Administration of Traditional Chinese Medicine issued the Notices on Establishing and Perfecting A Collaboration Mechanism of Traditional Chinese Medicine and Western Medicine for Epidemic Control including COVID-19 together, which regulated to establishing a collaboration mechanism of TCM and western medicine and increasing treatment powers of TCM. On February 13, 2020, the central government held a meeting of the leading group to cope with COVID-19 epidemics, which asked to combine TCM and western medicine, facilitate deep intervention of TCM throughout the treatment of patients with COVID-19, and promote effective prescriptions and Chinese patent medicine timely. However, there's a doubt about the participation of TCM in Wuhan, according to public reports. TCM was applied to intervention for the first time and achieved a high participation $(>90 \%)$ in many other provinces and cities. However, the $24^{\text {th }}$ conference of Hubei Province on COVID-19 epidemic control work announced on February 14, 2020, that the participation rate of TCM in treatment to diagnosed patients in was higher than $75 \%$ in relative to the national level of $85.2 \%(60,107$ cases) by February 17, 2020. On February 19, 2020, the National Health Commission of Hubei Province issued the Notices on Further Improving Cooperation of Traditional Chinese Medicine and Western Medicine in COVID-19 Epidemic Control, which emphasized on increasing participation and arrangement measures of TCM in the intervention. Hubei Province is not the sole province that makes delayed responses to the use of TCM. Currently, TCM still faces many barriers to participate in public health emergencies before the COVID-19 epidemic. As a legal scholar and a fan and a learner of Chinese traditional cultures, the author attempted to make an in-depth 
study and propose some legal countermeasures to promote the participation of TCM in various public health emergencies to save lives and protect people. The author also expects to make some contributions to the revitalization of TCM.

\section{Barriers}

The public health emergency system of the People's Republic of China was built up to cope with SARS epidemics in 2003. According to Regulation on the Urgent Handling of Public Health Emergencies (passes in 2003 and revised in 2010), public health emergencies refer to serious infectious disease epidemic, massive diseases of unknown causes, serious food and occupational poisoning which occur suddenly and can or might cause severe damages to public social health as well as other events that can cause serious impacts on public health. After 2003, TCM has presented outstanding performances in sudden events, including the Wenchuan Earthquake, Yushu Earthquake, Zhouqu Debris-flow Disaster, etc. However, TCM still has a limited role in response to public health emergencies, and it still faces many barriers to participate in public health emergencies.

Firstly, the medical system dominated by western medicine has natural institutional difficulties against the intervention of TCM.

Similar to the impact of university education mode from western countries on the old-style private schools in China, China finally chooses the medical system dominated by western medicine for practical reasons. According to public data, there were more than 500 hospitals of traditional Chinese and Western medicine in China by the end of 2017. Still, there are respectively more than 10,000 public hospitals and private hospitals in the same period. General hospitals have TCM departments, but they only occupy a very low proportion. Although there are no accurate data available yet, the low proportion of TCM hospitals and TCM department in the whole medical system is an indisputable fact. Such a low proportion is more prominent in Wuhan, the center of the COVID-19 epidemic. Wuhan ranks top among Chinese cities in terms of medical power for the settlement of many super-strong general hospitals, including Tongji Hospital, Concord Hospitals, People's Hospital of Wuhan University, and Central South Hospital. However, such an ultra-strong western medicine system brings greater inertial difficulties for intervention of TCM. This can be proved by screenshots of dialogues between medical workers who came to Wuhan for the COVID-19 epidemic fight on the Internet.

Secondly, the nonuniform setting of local supervision departments for TCM leads to the unbalanced development of TCM in China.

In the field of market failure, government supervision can influence the development of the field directly. The State Council of China set a National Administration of Traditional Chinese Medicine (NATCM), which is subordinate to the National Health Commission of the People's Republic of China. According to the function-organization-system fixation scheme, NATCM possesses not only macroscopic functions of "formulating strategies, plans and policies for the development of TCM and ethnic medicine industry," but also microscopic supervision responsibilities of "treatment, prevention, health care, rehabilitation and clinical administration of TCM." Due to the vast territory and abundant resources, China has unbalanced development among different regions, and settings of TCM administration bureaus vary in different regions. In view of links of 31 provinces (autonomous regions and municipalities) and Hong Kong 
Special Administrative Region provided by NATCM, only websites of TCM administration bureaus of Beijing, Jilin, Zhejiang, Anhui, Henan, Hunan, Tibet and Shaanxi are accessible. The author further checked official websites of TCM administration bureaus of 35 provinces (autonomous regions, municipalities, Xinjiang Production and Construction Corps, special administrative regions and Taiwan, subjected to the official website of the State Council), and found that only five provinces have set TCM administration bureaus, which are Shanxi Province, Fujian Province, Jiangxi Province, Guangdong Province and Guangxi Province. Among these official websites, some will skip automatically to the subordinate webpage of NATCM. With respect to information disclosure, which is an essential evaluation standard in existing reform of the administrative system, there are great regional differences in the setting of TCM supervision departments in China, which will surely lead to an unbalanced TCM development level. Accordingly, the response of TCM to emergencies is influenced.

Thirdly, the legal system of public health emergencies lacks regulation on the collaboration of TCM and western medicine.

Significant and direct legal references involved in medical emergency responses to COVID-19 epidemic include Law of the People's Republic of China on the Prevention and Treatment of Infectious Diseases (passed in 1989 and revised in 2004), Emergency Response Law of the People's Republic of China (passed in 2007), Regulation on the Urgent Handling of Public Health Emergencies and National Contingency Plan for Public Health Emergencies (issued in 2006). The whole of China entered into an emergency state after the occurrence of the COVID-19 epidemic. Provinces and cities set up a corresponding level of emergency response center. The department of health administration was responsible for "investigating, controlling, and medical saving issues," while other departments were held responsible for relevant tasks in their scopes of official duties. After the occurrence of the COVID-19 epidemic, the whole country from central government to the National Health Commission of the People's Republic of China, NATCM, and even the Health Commission of Hubei Province all have made great efforts in allocation and promotion of TCM intervention. Unfortunately, there are some problems in implementation. Under the current medical system, general hospitals centered in western medicine will surely occupy the leading role in emergency admission and treatment of patients. Under this circumstance, how to combine TCM and western medicine is a very complicated problem, for example, how to connect the infection department of traditional Chinese medicine and Western Medicine (Wang et al., 2020: 9). Based on reviewing of legal norms and policy documents at different levels, the author found no description of a combination of TCM and western medicine. Of course, it is understandable that there's no detailed legal regulation over the allocation of medical resource allocation. Nevertheless, no regulation on practices of combining TCM and western medicine has been formed yet in emergency plans at all levels.

Fourthly, there's a lack of reasonable public understanding of TCM and a dilemma of folk traditional Chinese doctor practice.

The delayed action of Hubei Province in using TCM intervention indicates that China has some barriers against the implementation of combining TCM and western medicines in response to public health emergencies. However, great contradictions of online public opinion over TCM, including superstition and mystification of TCM, strong boycott of TCM and neutral attitudes, imply a great dispute on TCM in China (Li, 2012: 80). To some extent, folk 
trust and supports to TCM might be more difficult to be achieved compared to official approval and promotion. It is the public that is the primary victims of public health emergencies. The participation effect of TCM in response to public health emergencies will be affected significantly if the public has no rational understanding of TCM.

How folk TCM doctors participate in public health emergencies is another challenge. During the COVID-19 epidemic, rejection of folk TCM doctors to "fight request" and private visits of folk TCM doctors are disclosed online continuously. Difficulties for folk TCM doctors to participate in responses to public health emergencies are manifested in the following aspects. Firstly, some folk TCM doctors either have no certificate or are facing mandatory shutdowns during the epidemic period, even though they have certificates or practice places. Secondly, folk TCM doctors scatter around, which brings difficulties in organization and allocation. Thirdly, although some therapies of folk TCM doctors are effective, they cannot be verified according to an existing associated standard system. In a word, the great power of folk TCM doctors still remains underused in public health emergencies.

\section{Countermeasures}

The important assistance has proved the necessity of TCM in response to public health emergencies during SARS and curative effect in some aspects of the COVID-19 epidemic (Huang, 2020: 2). However, there are still many barriers against its participation in public health emergencies, regardless of the great official promotion efforts. With considerations to the achievements and problems of TCM in COVID-19 epidemic, the author proposed some immature policy suggestions in the light of limited experiences:

1. Perfect legal regulations concerning the combination of TCM and western medicine in responses to public health emergencies. National Contingency Plan for Public Health Emergencies is composed of general rules, system functions, administration guarantee, and emergency plan management supplementary provisions. With respect to the working principle, statements to protect and strengthen the participation of TCM in public health emergencies can be supplemented. In administration guarantee, contents about the allocation of TCM hospitals and TCM teams, as well as a combination of TCM and western medicines, can be added into the emergency medical treatment team and maneuver. Considering unique situations, China issued the Law of Traditional Chinese Medicine in 2016 to inherit and promote TCM and facilitate TCM industrial development. Article 18 of Law of Traditional Chinese Medicine regulates explicitly that People's Government of county-level or higher shall make full use of TCM in responses to public health emergencies and strengthen emergency supplies, equipments, facilities, technologies, and talent resources of TCM. However, there's no description of the combination of TCM and western medicine in this regulation, which is the essential key in emergency practices (Yang \& Zhang, 2013: 152). Therefore, supplement provision shall be discussed and given. Moreover, attention shall be paid to how to implement legal responsibilities rather than a mere scrap of paper. For example, the first article of legal responsibility in Law of Traditional Chinese Medicine stipulates that "TCM administration sectors and other sectors of People's Government of county-level or higher are instructed by People's Government at the same level or higher levels to correct for non-fulfillment of relevant responsibilities; persons in charge and persons directly responsible will be punished according to laws in cases of gross violation." From this perspective, whether the People's Government of county-level or higher can be held responsible for non-action in strengthening 
TCM intervention in responses to public health emergencies? How? (Su et al., 2019: 16651668)

2. Improve the position of local TCM sectors and strengthen the promotion of TCM development. Why is it necessary to set up a TCM administration bureau since there's the National Health Commission of the People's Republic of China? Why is it necessary to issue the Law of Traditional Chinese Medicine as there are Law of Practicing Physicians and Regulations on Medical Organization Administration? Reflection on these problems is of important significance in the background of the strong role of western medicine and the weak role of TCM since the foundation of the People's Republic of China. Deng Tietao, a TCM master in the first session, recalled that due to great endeavors of the old generation of TCM doctors in the 1980s, the central government decided to set a National Ministry of Traditional Chinese Medicine on the level of State Council for special management of TCM affairs. The Regulations on Traditional Chinese Medicine, which was issued by the State Council in 2003, cannot adapt to new problems against TCM industrial development. Therefore, the Law of Traditional Chinese Medicine has developed accordingly. Special management and legislation of TCM have to be set up because TCM is the field that is easier to suffer market failure compared to western medicine, and there are great differences in national planning, training, and supervision of TCM (Su, 2015: 2). In particular, the competent department of the local government is extremely important. Attentions of central government often will end up with significant differences in local execution powers after delivery through several levels. The author suggested that the provincial government shall set uniform ministry of TCM administration in China, and existing structures of TCM in department and organizations shall be adjusted after beating the COVID-19 epidemic. Ministry of TCM Administration at the city level and county level shall be set according to local situations. According to situations in some provinces and cities, some provinces have no ministry of TCM administration, but many cities of the province have set ministries of TCM administration. In January 2019, the first Ministry of TCM Administration at the county level was established officially in Zhejiang Province, which was also one of few special TCM administration organizations at the county level in China.

3. Expand fields of TCM in response to public health emergencies. During the COVID-19 epidemic, the public's attention on TCM still focuses on centralized treatment at designated hospitals and the cooperation of western medicine. In fact, it has stipulated in the Law of Traditional Chinese Medicine that TCM has more application fields in public health emergencies: "medical and health organizations shall take the initiative to use TCM theories and techniques in disease prevention and control" (Article 18). TCM owns unique advantages in disease prevention. Various protection measures are indispensable to medical staff who are engaged in treatment at designated hospitals. However, whether additional protection can be offered to them by taking TCM or other TCM treatment means? Except for designated hospitals, TCM can play an important role in disease prevention at quarantine sites (Cao, 2013: 3). Clustering infection in nursing homes, mental hospitals, and prisons have been reported during the COVID-19 epidemic, which gave us an important lesson. At present, the fight against the COVID-19 epidemic is going to win, and China is facing challenges for return to work and school. Enterprises and organizations can implement prevention measures by making full use of stifling, perfumed medicine bags, and oral taking of TCM. Additionally, the role of TCM in treatment to patients with severe and critical disease conditions deserves enough attention. Zhang Boli, an academician of the Chinese Academy of Engineering and 
the president of Tianjin University of Traditional Chinese Medicine, talked in the interview of CCTV that he was exploring a precipitation with Professor Liu Qingquan, the director of Beijing Chinese Medicine Hospital. They have participated in the treatment of three patients in critical condition (all three patients were doctors) and saved them from death. Similar cases are taken place continuously in Wuhan. Such exciting news is enough to break the public's opinion on "treatment to chronic diseases" and "slow progress" of TCM (Cao, 2020: 2). What are other fields that TCM can participate in? This shall be studied and demonstrated by enlisting TCM into the institutional program and then writing into the relevant emergency plan.

4. Open channels for folk TCM doctors to participate in responses to public health emergencies. In the whole response process to public health emergencies, public medical institutions assume the main force of admission and treatment of patients. Based on the response to the COVID-19 epidemic, medical reinforcements are mainly from public medical institutions. However, there's a considerable power of scattered folk TCM doctors in China. Opening channels for folk TCM doctors to participate in public health emergencies can relieve the shortage of medical resources significantly. In fact, cross-infection after massive admission of patients with fever into hospital is one of the causes of the serious epidemic in Wuhan. Mild cases occupy a high proportion of such public health emergency of virus-induced respiratory infection, and there are objective conditions for scattered treatment and even remote diagnosis and treatment. Since medical institutions where folk TCM doctors have small scales and potential risk in prevention and disinfection, remote diagnosis and treatment can be considered. The rapid development of online medical services in recent years provides strong technological and market mechanism supports for establishing an online medical treatment pathway after the occurrence of epidemics. During responses to public health emergencies, an online remote diagnosis and treatment platform for folk TCM doctors shall be established by encouraging enterprises to provide free services or at the cost of government. Of course, only folk TCM doctors with relevant certificate can apply for remote diagnosis and treatment. TCM doctors must pass the assessment before practices, which is extensively denounced by advocators in the long period. TCM advocators believe that different from western medicine, TCM certificate shall be given according to practical effect rather than assessment. The author deemed that in 2017, the original Interim Measures for Assessment and Registration Management of Certificates to Traditional Chinese Medicine Doctors with Specialty issued by National Health and Family Planning Commission of the People's Republic of China-proposed regulation on assessment and practice registration to doctors who have learned TCM from masters or practiced over the years and have a specialty. This solves the long-term problem of legal practices of folk TCM doctors. On the contrary, folk TCM doctors who have not gained certificates according to these interim measures are judged illegal practice of medicines even though they have practical medical ability and level, and they cannot be recruited to respond team to public health emergencies (Deng, 2017: 2).

5. Strengthen promotion and guide public opinion to help the public form a rational understanding of TCM. The modern concept of "TCM" is originated from modern times. For distinguishing from the introduced western medicines before and after the First Opium War, the name of traditional Chinese medicine was proposed. In 1936, Regulations of Traditional Chinese Medicines, which were formulated by the Kuomintang Government determined the legal term of "traditional Chinese medicine." However, TCM took a back seat as western medicine became the main medical power in the society. More and more medical instruments and drugs were derived from the western medicine diagnosis and treatment technologies, 
which involved huge market benefits. Since then, TCM and western medicine began to form competition and opposite relations. It is widely accepted that the benefit group of western medicine is to slander and shame the backbone force of TCM. Another group opposes TCM for doubts on some theories and practices of TCM, which disagree with the spirit of modern sciences. Opposite with "shaming TCM," some "TCM fans" are blind in believing TCM and exaggerate the curative effect (of course, there might be some stakeholders). The Layout for Traditional Chinese Medicine Development Strategic Planning (2016-2030), which was published by the State Council, gave a special statement on public opinion and propaganda. It expects that "a good pattern for the thick atmosphere of "trusting TCM, loving TCM and using TCM' and joint development of TCM" can be formed in the whole society. Public are extremely sensitive to various diagnosis and treatment schemes and information at the occurrence of public health emergencies. Rational understanding of the public on TCM is fundamental to give full play of TCM in response to public health emergencies. Therefore, rational popularization guidance on public opinion through various modes, especially through new media and self-media, is needed in addition to the front-line treatment during responses to public health emergencies.

\section{Conclusions}

It is confident that TCM will win more and more trusts after the COVID-19 epidemic. Moreover, emergency treatment during the COVID-19 epidemic also accumulates valuable experiences for TCM to participate in public health emergencies. The author hopes that the performance and problems of TCM in the COVID-19 epidemic can attract enough attention. It is expected to perfect and strengthen relevant legal system and government supervision ability and supplement regulations on a combination of TCM and western medicine and TCM intervention fields at the appropriate time to protect participation of TCM in public health emergencies. Finally, the author cited the opinion of Zhang Boli on the fight between TCM and western medicine: there's no need to fight between TCM and western medicine because both are for disease treatment, and they have unique advantages. Chinese people shall be pleased to have two medical systems of TCM and western medicine.

\section{미 References}

Cao, Hongxin (2020) Making full use of advantages of traditional Chinese medicine in epidemic control. Guangming Daily, February 12.

Cao, Hongxin (2013) In response to sudden infectious diseases, traditional Chinese medicine should do something, China Journal of traditional Chinese medicine, August 14.

Deng, Yong (2017) Legislation to ensure the practice of folk traditional Chinese medicine, China Journal of traditional Chinese medicine, January 1.

Huang, Luqi (2020) Promotion of development of traditional Chinese medicine through peacetime and wartime uses. Study Times, March 23.

Li, Yongping (2012) Agenda setting and public opinion guidance of Journals - also on the academic value of Past Events of Traditional Chinese Medicine 1910-1949, Research on Publication and Distribution, December 15.

Liang, Xiao (2020) Fight against "Epidemics" shall make a good use of the weapon of traditional Chinese medicine. Shanxi Daily, April 9.

$\mathrm{Su}$, Qingmin (2015) Chinese medicine supervision needs top-level system ice breaking, China 
Journal of traditional Chinese medicine, January 22.

$\mathrm{Su}$, Rui, Guo Yuhong, and Liu Qingquan (2019) Research on the current status of emergency response of traditional Chinese medicine and relevant policies. Journal of Emergency in Traditional Chinese Medicine, September 15.

Wang, Dongbo, Yin Zheng, Chen Weizhen, Bai Yongmei, and Han Demin (2020) Current situation of hospital infection emergency management in China's public health emergency system, China hospital management, April 5.

Yang, Jing, and Zhang Zhongyuan (2013) Research on the mechanism of Chinese medicine participating in public emergency response based on management level, China Health Administration, February 15. 